\title{
Pulmonary Atresia with Intact Ventricular Septum Causing Severe Left Ventricular Outflow Tract Obstruction
}

\author{
Payal Amin · Daniel S. Levi • Maggie Likes • \\ Hillel Laks
}

Received: 12 January 2009/ Accepted: 16 March 2009/Published online: 14 April 2009

(c) The Author(s) 2009. This article is published with open access at Springerlink.com

\begin{abstract}
We describe an infant with pulmonary atresia with intact ventricular septum (PAIVS) and severe left ventricular outflow tract (LVOT) obstruction secondary to a suprasystemic right ventricle causing leftward displacement of the interventricular septum. Imaging demonstrated an aneurysmal dilation at the base of the proximal main pulmonary artery (MPA) with no forward flow from the right ventricle. During transannular patch and central shunt placement, the communication between the pulmonary artery and the right ventricle was enlarged to ensure adequate decompression. We report this successful palliation and resulting complete elimination of the LVOT obstruction in a very unique presentation of PAIVS in a newborn.
\end{abstract}

Keywords CHD - Valve lesions - Aneurysm ·

Myocardial · Pulmonary valve

\section{Introduction}

Pulmonary atresia with intact ventricular septum (PAIVS) is a congenital cardiac defect with a broad spectrum of anatomic variability $[4,5]$. Although the incidence of PAVIS is very low (according to Daubeney et al. [2], 4.5 in 100,000 live births), the incidence of PAIVS with any

P. Amin · D. S. Levi $(\bowtie) \cdot$ M. Likes

Division of Pediatric Cardiology, Mattel Children's Hospital at UCLA, Los Angeles, CA 90095-1743, USA

e-mail: DLevi@ucla.edu

P. Amin

e-mail:pamin01@gmail.com

H. Laks

Division of Cardiothoracic Surgery, Mattel Children's Hospital at UCLA, Los Angeles, CA 90095-1743, USA impediment of the left ventricular outflow tract (LVOT) is even less frequently seen, as low as 4 in 183 patients born with PAIVS [1].

In the following case study, we describe a patient with PAIVS that resulted in severe LVOT obstruction from an aneurysmal right ventricle (RV) bowing leftward as a result of suprasystemic RV pressures. This patient also had a very unique aneurysm of the right ventricular outflow tract (RVOT) with only limited communication with the RV. $\mathrm{RV}$ decompression with a transannular patch placement off cardiopulmonary bypass (CPB) resulted in complete relief of the LVOT obstruction. To our knowledge, this is the first report of LVOT obstruction by an RV in a patient with PAIVS.

\section{Case Report}

An infant girl of estimated gestational age 37 weeks, weighing $2.9 \mathrm{~kg}$, was born via normal spontaneous vaginal delivery. On initial exam, the patient was found to be cyanotic with an oxygen saturation of $70 \%$. The patient was intubated and started on prostaglandin $\mathrm{E}_{1}$.

Two-dimensional echocardiography showed PAIVS with a severely hypoplastic tricuspid valve $(z$-score $<-4)$. A small, hypertrophied RV was visualized with an aneurysmal interventricular membranous septum obstructing the LVOT, producing flow turbulence and a peak instantaneous gradient of $77 \mathrm{mmHg}$ by echocardiography. A small nonrestrictive patent foramen ovale (shunting right-to-left), and confluent pulmonary arteries supplied by a tortuous patent ductus arteriosus (PDA) were also noted. The left ventricle (LV) was not severely hypertrophied (Fig. 1a).

A successful balloon atrial septostomy was performed on day of life one. Cardiac catheterization confirmed the 
Fig. 1 a Two-dimensional echocardiogram, subcostal view (preoperative), showing severe LVOT obstruction (arrow). $\mathrm{AV}=$ aortic valve, $\mathrm{MV}=$ mitral valve. $\mathbf{b}$ Twodimensional apical four chamber echocardiogram (postoperative) demonstrates an unobstructed LVOT (arrow)
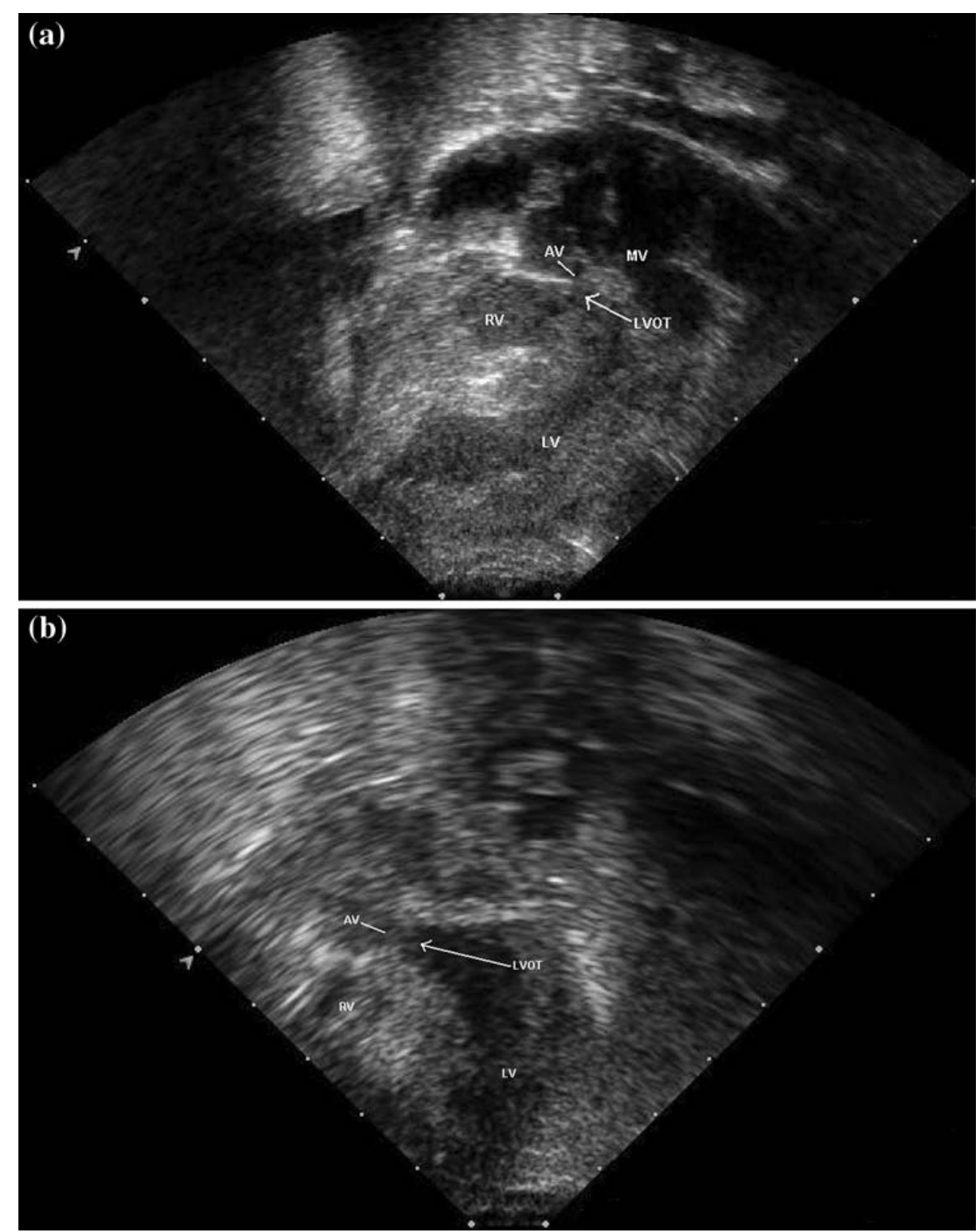

echocardiographic findings (Fig. 2). A RV injection demonstrated no antegrade flow into the pulmonary artery (PA). Pressure recordings done during the catherization showed a peak-to-peak LVOT gradient of $50 \mathrm{mmHg}$. Coronary angiography revealed normal anatomy without RV-dependent coronary circulation (RVDCC) and normal caliber confluent branch pulmonary arteries. Magnetic resonance imaging was also performed and confirmed the presence of a septated $\mathrm{RV}$, consisting of a larger dorsal chamber and a smaller anterior chamber. The larger posterior chamber had mural hypertrophy and showed no evidence of antegrade flow. This chamber exerted a masstype effect, bulging the interventricular septum toward the left side with resultant compressive effects on the LVOT (Fig. 3).
Surgical palliation was performed on day of life four without the use of CPB. Direct measurement of RV pressures revealed an $\mathrm{RV}$ pressure $10 \mathrm{mmHg}$ greater than the aortic pressure. A transannular patch of glutaraldehydetreated pericardium was prepared and sutured from the base of the aneurysmal dilation to the pulmonary bifurcation distally. A membrane was identified on the left inferior aspect of the aneurysmal dilation. This membrane completely obstructed the RV outflow during systole. A rhizotomy knife was used to enlarge this communication until pulsatile forward flow was noted in the pulmonary arteries. Transesophageal echocardiogram and direct pressure measurements performed intraoperatively showed that the $\mathrm{RV}$ pressure immediately fell to approximately one-half systemic with the excision of the flap valve. Additionally, 


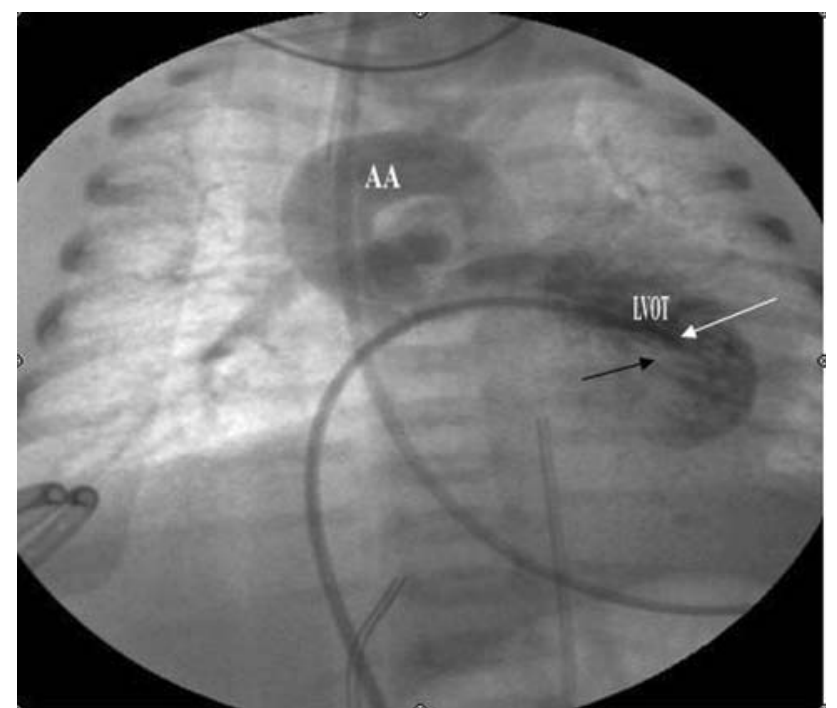

Fig. 2 This cineangiogram (preoperative) shows the catheter tip (white arrow) in the hypertrophied LV. Note the RV impinging on the LVOT (black arrow). AA $=$ aortic arch

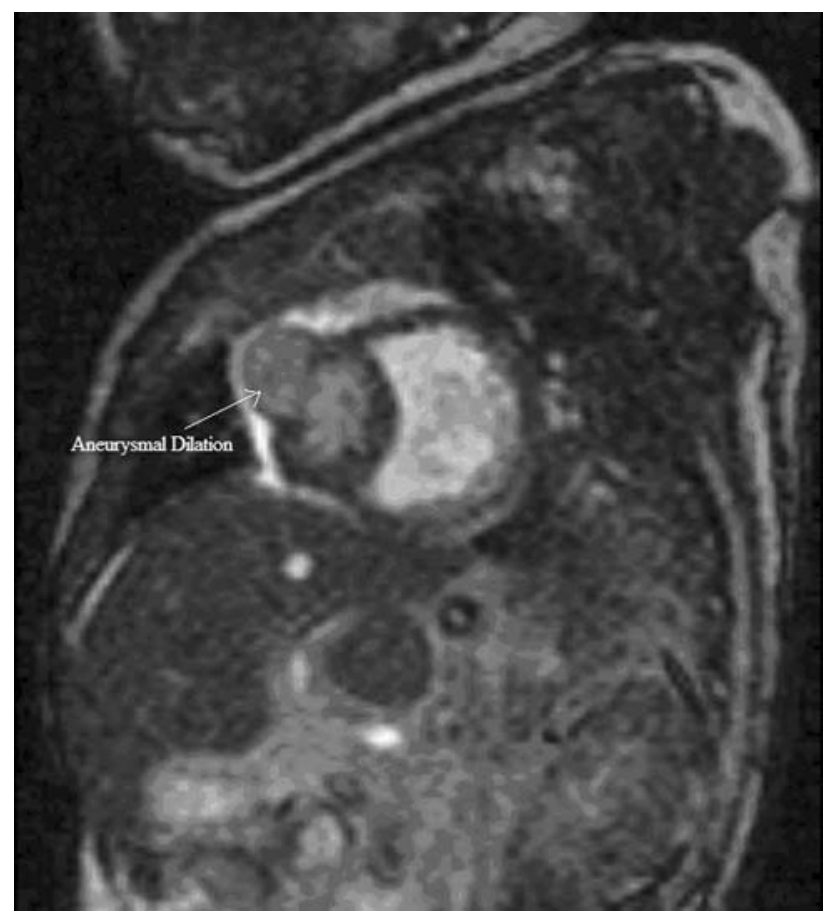

Fig. 3 Short-axis magnetic resonance image that demonstrates an aneurysmal dilation in the RV

the tricuspid valve was noted to have begun functioning with forward flow into the RV. The RV pressure continued to decrease to less than one-half systemic. The pressures in the left ventricle (LV) and aorta equalized. Because occlusion of the PDA resulted in inadequate pulmonary blood flow, a 4-mm central shunt of reinforced Gore-Tex was placed. Using a Doppler probe, it was found that on occluding the shunt, there was pulsatile forward flow from the RV, and on opening the shunt, there was continuous flow as well.

The infant tolerated the surgery without complications. She was weaned off the prostaglandin overnight. A postoperative echocardiogram done the following day demonstrated a patent LVOT (measuring 4-5 mm), with a peak gradient of $12 \mathrm{mmHg}$, significantly lower than the preoperative echocardiogram $(77 \mathrm{mmHg}$ ) (Fig. 1b). The patient is currently doing well and has since undergone a Glenn shunt placement and atrial septectomy for tricuspid hypoplasia.

\section{Discussion}

This case demonstrates a patient with PAIVS who had two very unique features: (1) LVOT obstruction secondary to aneurysmal outpouching of the membranous interventricular septum secondary to suprasystemic RV pressures and (2) RVOT obstruction secondary to an internal membrane separating the RV body from the RVOT aneurysm. An offpump transannular patch operation was used to ameliorate both problems by excising the RVOT membrane and decompressing the RV to relieve the LVOT obstruction. The success of the operation was possible because of the lack of RVDCC.

The variation in anatomy of PAIVS determines the course of treatment in each patient. Because our patient had normal coronary anatomy without RVDCC, RV decompression via outlet enlargement and anatomic reinstitution of an RV-pulmonary artery connection was possible and ideal. Decompression of the RV in patients has been shown to relieve the RV hypertension, reduce tricuspid regurgitation allowing for forward flow, and potentiate the growth of both the tricuspid valve annulus and the RV itself [5]. In this patient, surgical relief of RV hypertension, by placement of a patch and excision of the internal membrane, resulted in reestablishment of tricuspid valve function and completely relieved LVOT obstruction. Of note, this internal membrane, which caused RVOT obstruction and likely contributed to the LVOT obstruction, has not been previously reported and is unique to our patient. Although transcather palliation of this lesion might have been possible, we used a surgical approach because of the complex RVOT anatomy and because there appeared to be a significant distance between the main pulmonary artery (MPA) and RV. If this type of anatomy is seen in the future, magnetic resonance imaging will be essential prior to attempts to create antegrade flow with catheter-based techniques.

Due to the hypoplastic nature of this patient's RV, surgical decompression alone did not provide pulmonary blood flow adequate for appropriate arterial oxygen 
saturations. Previous studies have shown that valvotomy alone often fails to maintain adequate saturations secondary to reduced RV compliance; thus placement of a systemic-to-pulmonary shunt is often indicated. This was the case for the patient described $[3,5]$.

In patients with PAIVS and secondary LVOT obstruction from RV hypertension, expeditious RV decompression can relieve LVOT obstruction. This is clearly only possible if there is relatively normal coronary circulation without RVDCC. This case also demonstrates that an obstructed, aneurismal RVOT/PA can be remedied without CPB. Our patient had a very favorable outcome despite her very unusual anatomy.

Open Access This article is distributed under the terms of the Creative Commons Attribution Noncommercial License which permits any noncommercial use, distribution, and reproduction in any medium, provided the original author(s) and source are credited.

\section{References}

1. Daubeney PE, Delany DJ, Anderson RH et al (2002) Pulmonary atresia with intact ventricular septum. Range of morphology in a population-based study. J Am Coll Cardiol 39(10):1670-1679

2. Daubeney PE, Sharland GK, Cook AC et al (1998) Pulmonary atresia with intact ventricular septum. Impact of fetal echocardiography on incidence at birth and postnatal outcome. Circulation 98:562-566

3. De Leval M, Bull C, Stark J, Anderson RH, Taylor JFN, Macartney FJ (1982) Pulmonary atresia and intact ventricular septum: surgical management based on a revised classification. Circulation 66(2):272-280

4. Galindo A, Drant SE (2001) Pulmonary atresia with intact ventricular septum (PA/IVS) diagnostic and interventional cardiac catherization in the neonate. Prog Pediatr Cardiol 13:177-182

5. Laks H, Plunkett MD (2001) Surgical management of pulmonary atresia with intact ventricular septum. Prog Pediatr Cardiol 13:183-197 